\title{
Contact CR-submanifolds of an indefinite Lorentzian para-Sasakian manifold
}

\author{
Barnali Laha \\ Jadavpur University \\ Department of Mathematics \\ Kolkata-700032, India \\ email: barnali.laha87@gmail.com
}

\author{
Bandana Das \\ Jadavpur University \\ Department of Mathematics \\ Kolkata-700032, India \\ email: badan06@yahoo.co.in
}

\author{
Arindam Bhattacharyya \\ Jadavpur University \\ Department of Mathematics \\ Kolkata-700032, India \\ email: bhattachar1968@yahoo.co.in
}

\begin{abstract}
In this paper we prove some properties of the indefinite Lorentzian para-Sasakian manifolds. Section 1 is introductory. In Section 2 we define $\mathrm{D}$-totally geodesic and $\mathrm{D}^{\perp}$-totally geodesic contact $\mathrm{CR}$ submanifolds of an indefinite Lorentzian para-Sasakian manifold and deduce some results concerning such a manifold. In Section 3 we state and prove some results on mixed totally geodesic contact CR-submanifolds of an indefinite Lorentzian para-Sasakian manifold. Finally, in Section 4 we obtain a result on the anti-invariant distribution of totally umbilic contact CR-submanifolds of an indefinite Lorentzian para-Sasakian manifold.
\end{abstract}

\section{Introduction}

Many valuable and essential results were given on differential geometry with contact and almost contact structure. In 1970 the geometry of cosymplectic

2010 Mathematics Subject Classification: 53C15, 53C21, 53C25, 53C50

Key words and phrases: contact CR-submanifold, totally umbilic submanifold, indefinite Lorentzian para-Sasakian manifold, $\mathrm{D}$-totally geodesic, $\mathrm{D}^{\perp}$-totally geodesic, mixed totally geodesic, anti invariant distribution $\mathrm{D}^{\perp}$ 
manifold was studied by G. D. Ludden [14]. After them, in 1973 and 1974, B. Y. Chen and K. Ogive introduced the geometry of submanifolds and totally real submanifolds in [8], [17], [7]. Then K. Ogive expressed the differential geometry of Kaehler submanifolds in [17]. In 1976 contact manifolds in Riemannian geometry were discussed by D. E. Blair [5]. Later on, A. Bejancu discussed CRsubmanifolds of a Kaehler manifold [1], [2], [4], and then, K. Yano and M. Kon gave the notion of invariant and anti invariant submanifold in [13] and [21]. M. Kobayashi studied CR-submanifolds of a Sasakian manifold in 1981 [12]. New classes of almost contact metric structures and normal contact manifold in [18], [6] were studied by J. A. Oubina, C. Calin and I. Mihai. A. Bejancu and K. L. Duggal introduced ( $\epsilon$ )-Sasakian manifolds. Lightlike submanifold of semi Riemannian manifolds was introduced by K. L. Duggal and A. Bejancu [10], [9]. In 2003 and 2007, lightlike submanifolds and hypersurfaces of indefinite Sasakian manifolds were introduced [11]. Lastly, LP-Sasakian manifolds were studied by many authors in [15], [16], [19], [20].

In this paper we define D-totally and $\mathrm{D}^{\perp}$ - totally geodesic contact CRsubmanifolds of an indefinite Lorentzian para-Sasakian manifold and prove some interesting results.

An n-dimensional differentiable manifold is called indefinite Lorentzian paraSasakian manifold if the following conditions hold

$$
\begin{aligned}
& \phi^{2} X=X+\eta(X) \xi, \quad \eta \circ \phi=0, \quad \phi \xi=0, \quad \eta(\xi)=1, \\
& \tilde{g}(\phi X, \phi Y)=\tilde{g}(X, Y)-\epsilon \eta(X) \eta(Y), \\
& \tilde{g}(X, \xi)=\epsilon \eta(X),
\end{aligned}
$$

for all vector fields $X, Y$ on $\tilde{M}[5]$ and where $\epsilon$ is 1 or -1 according to $\xi$ is space-like or time-like vector field.

An indefinite almost metric structure $(\phi, \xi, \eta, \tilde{g})$ is called an indefinite Lorentzian para-Sasakian manifold if

$$
\left(\tilde{\nabla}_{X} \phi\right) Y=g(X, Y) \xi+\epsilon \eta(Y) X+2 \epsilon \eta(X) \eta(Y) \xi,
$$

where $\tilde{\nabla}$ is the Levi-Civita $(\mathrm{L}-\mathrm{C})$ connection for a semi-Riemannian metric g. Also we have

$$
\tilde{\nabla}_{X} \xi=\epsilon \phi X
$$

where $\mathrm{X} \in \mathrm{T} \tilde{\mathrm{M}}$.

From the definition of contact CR-submanifolds of an indefinite Lorentzian para-Sasakian manifold we have 
Definition 1 An $\mathrm{n}$-dimensional Riemannian submanifold $\mathrm{M}$ of an indefinite Lorentzian para-Sasakian manifold $\tilde{M}$ is called a contact CR-submanifold if

i) $\xi$ is tangent to $\mathrm{M}$,

ii) there exists on $\mathrm{M}$ a differentiable distribution $\mathrm{D}: \mathrm{x} \longrightarrow \mathrm{D}_{\mathrm{x}} \subset \mathrm{T}_{\mathrm{x}}(\mathrm{M})$, such that $\mathrm{D}_{\mathrm{x}}$ is invariant under $\phi$; i.e., $\phi \mathrm{D}_{\mathrm{x}} \subset \mathrm{D}_{\mathrm{x}}$, for each $\mathrm{x} \in$ $\mathrm{M}$ and the orthogonal complementary distribution $\mathrm{D}^{\perp}: \mathrm{x} \longrightarrow \mathrm{D}_{\mathrm{x}}^{\perp} \subset$ $\mathrm{T}_{x}{ }^{\perp}(\mathrm{M})$ of the distribution $\mathrm{D}$ on $\mathrm{M}$ is totally real; i.e., $\phi \mathrm{D}_{\mathrm{x}}^{\perp} \subset \mathrm{T}_{\bar{x}}^{\perp}(\mathrm{M})$, where $\mathrm{T}_{\mathbf{x}}(\mathrm{M})$ and $\mathrm{T}_{\mathrm{x}}^{\perp}(\mathrm{M})$ are the tangent space and the normal space of $M$ at $x$.

$\mathrm{D}\left(\right.$ resp. $\left.\mathrm{D}^{\perp}\right)$ is the horizontal (resp. vertical) distribution. The contact CRsubmanifold of an indefinite Lorentzian para-Sasakian manifold is called $\xi$ horizontal (resp. $\xi$-vertical) if $\xi_{x} \in \mathrm{D}_{\mathrm{x}}\left(\right.$ resp. $\xi_{\mathrm{x}} \in \mathrm{D}_{\mathrm{x}}^{\perp}$ ) for each $\mathrm{x} \in \mathrm{M}$ by [12].

The Gauss and Weingarten formulae are as follows

$$
\begin{aligned}
& \tilde{\nabla}_{X} Y=\nabla_{X} Y+h(X, Y), \\
& \tilde{\nabla}_{X} N=-A_{N} X+\nabla_{X}^{\perp} N,
\end{aligned}
$$

for any $\mathrm{X}, \mathrm{Y} \in \mathrm{TM}$ and $\mathrm{N} \in \mathrm{T}^{\perp} \mathrm{M}$, where $\nabla^{\perp}$ is the connection on the normal bundle $T^{\perp} M, h$ is the second fundamental form and $A_{N}$ is the Weingarten map associated with $\mathrm{N}$ via

$$
g\left(A_{N} X, Y\right)=g(h(X, Y), N)
$$

The equation of Gauss is given by

$$
\tilde{R}(X, Y, Z, W)=R(X, Y, Z, W)+g(h(X, Z), h(Y, W))-g(h(X, W), h(Y, Z)),
$$

where $\tilde{R}$ (resp. R) is the curvature tensor of $\tilde{M}$ (resp. $M$ ).

For any $x \in M, X \in T_{\chi} M$ and $N \in T_{\chi}^{\perp} M$, we write

$$
\begin{aligned}
& X=P X+Q X \\
& \phi N=B N+C N,
\end{aligned}
$$

where PX (resp. BN) denotes the tangential part of $X($ resp. $\phi N)$ and QX (resp. $\mathrm{CN}$ ) denotes the normal part of $\mathrm{X}$ (resp. $\phi \mathrm{N}$ ) respectively. 
Using (6), (7), (10), (11) in (4) after a brief calculation we obtain on comparing the horizontal, vertical and normal parts

$$
\begin{aligned}
& \mathrm{P} \nabla_{X} \phi P Y-P A_{\phi Q Y} X=\phi P \nabla_{X} Y+g(P X, Y) \xi+\epsilon \eta(Y) P X+2 \epsilon \eta(Y) \eta(X) \\
& Q \nabla_{X} \phi P Y+Q A_{\phi Q Y} X=B h(X, Y)+g(Q X, Y) \xi+\epsilon \eta(Y) Q X \\
& h(X, \phi P Y)+\nabla_{X}^{\perp} \phi Q Y=\phi Q \nabla_{X} Y+C h(X, Y) .
\end{aligned}
$$

From (5) we have

$$
\begin{aligned}
& \nabla_{X \xi}=\epsilon \phi P X, \\
& h(X, \xi)=\epsilon \phi Q X .
\end{aligned}
$$

Also we have

$$
\begin{aligned}
& h(X, \xi)=0 \quad \text { if } \quad X \in D, \\
& \nabla_{X} \xi=0, \\
& h(\xi, \xi)=0, \\
& A_{N} \xi \in D^{\perp} .
\end{aligned}
$$

\section{D-totally geodesic and $\mathrm{D}^{\perp}$-totally geodesic con- tact CR-submanifolds of an indefinite Lorentzian para-Sasakian manifold}

First we define the D-totally (resp. $\mathrm{D}^{\perp}$-totally) geodesic contact CR-submanifold of an indefinite Lorentzian para-Sasakian manifold.

Definition 2 A contact CR-submanifold $\mathrm{M}$ of an indefinite Lorentzian paraSasakian manifold $\tilde{M}$ is called $\mathrm{D}$-totally geodesic (resp. $\mathrm{D}^{\perp}$-totally geodesic) if $\mathrm{h}(\mathrm{X}, \mathrm{Y})=0, \forall \mathrm{X}, \mathrm{Y} \in \mathrm{D} \quad\left(\right.$ resp. $\left.\mathrm{X}, \mathrm{Y} \in \mathrm{D}^{\perp}\right)$.

From the above definition, the following propositions follow immediately.

Proposition 1 Let $\mathrm{M}$ be a contact CR-submanifold of an indefinite Lorentzian para-Sasakian manifold. Then $\mathrm{M}$ is a D-totally geodesic if and only if $A_{\mathrm{N}} \mathrm{X} \in \mathrm{D}^{\perp}$ for each $\mathrm{X} \in \mathrm{D}$ and $\mathrm{N}$ a normal vector field to $\mathrm{M}$.

Proof. Let $M$ be D-totally geodesic. Then from (8) we get

$$
g(h(X, Y), N)=g\left(A_{N} X, Y\right)=0 .
$$


So if

$$
h(X, Y)=0, \quad \forall X, Y \in D
$$

i.e.,

$$
A_{N} X \in D^{\perp} .
$$

Conversely, let $A_{N} X \in D^{\perp}$. Then for $X, Y \in D$ we can obtain

$$
g\left(A_{N} X, Y\right)=0=g(h(X, Y), N)
$$

i.e.,

$$
h(X, Y)=0
$$

$\forall X, Y \in D$, which implies that $M$ is D-totally geodesic. Thus our proof is complete.

Proposition 2 Let $\mathrm{M}$ be a contact CR-submanifold of an indefinite Lorentzian para-Sasakian manifold $\tilde{\mathrm{M}}$. Then $\mathrm{M}$ is $\mathrm{D}^{\perp}$-totally geodesic if and only if $\mathrm{A}_{\mathrm{N}} \mathrm{X} \in \mathrm{D}$ for each $\mathrm{X} \in \mathrm{D}^{\perp}$ and $\mathrm{N}$ a normal vector field to $\mathrm{M}$.

Proof. The proof follows immediately from the above proposition.

Concerning the integrability of the horizontal distribution $\mathrm{D}$ and vertical distribution $\mathrm{D}^{\perp}$ on $\mathrm{M}$, we can state the following theorem:

Theorem 1 Let $M$ be a contact CR-submanifold of an indefinite Lorentzian para-Sasakian manifold. If $\mathrm{M}$ is $\xi$-horizontal, then the distribution $\mathrm{D}$ is integrable iff

$$
h(X, \phi Y)=h(\phi X, Y)
$$

$\forall \mathrm{X}, \mathrm{Y} \in \mathrm{D}$. If $\mathrm{M}$ is $\xi$-vertical then the distribution $\mathrm{D}^{\perp}$ is integrable iff

$$
A_{\phi X} Y-A_{\phi Y} X=\epsilon[\eta(Y) X-\eta(X) Y]
$$

$\forall X, Y \in D^{\perp}$.

Proof. If $M$ is $\xi$-horizontal, then using (14) we get

$$
h(X, \phi P Y)=\phi Q \nabla_{X} Y+C h(X, Y)
$$

$\forall X, Y \in D$. Therefore $[X, Y] \in D \quad$ iff $h(X, \phi Y)=h(Y, \phi X)$

Hence, if $M$ is $\xi$-horizontal, $[X, Y] \in D$ iff $h(X, \phi Y)=h(\phi X, Y)$. 
Again using (14) we get

$$
\nabla_{\mathrm{X}}^{\perp} \phi \mathrm{Y}=\mathrm{Ch}(\mathrm{X}, \mathrm{Y})+\phi \mathrm{Q} \nabla_{\mathrm{X}} \mathrm{Y}
$$

for $X, Y \in D^{\perp}$.

After some calculations we see that

$$
\begin{aligned}
\tilde{\nabla}_{X} \phi Y= & g(X, Y) \xi+\epsilon \eta(Y) X+2 \epsilon \eta(Y) \eta(X) \xi+\phi P \nabla_{X} Y \\
& +\phi Q \nabla_{X} Y+B h(X, Y)+C h(X, Y) .
\end{aligned}
$$

Again from (7) and (24) we get

$$
\begin{aligned}
\nabla_{X}^{\perp} \phi Y= & A_{\phi Y} X+g(X, Y) \xi+\epsilon \eta(Y) X+2 \epsilon \eta(Y) \eta(X) \xi \\
& +\phi P \nabla_{X} Y+\phi Q \nabla_{X} Y+B h(X, Y)+C h(X, Y)
\end{aligned}
$$

for $\mathrm{X}, \mathrm{Y} \in \mathrm{D}^{\perp}$. From (24) and (25) we can write

$$
\phi P \nabla_{X} Y=-A_{\phi Y} X-g(X, Y) \xi-\epsilon \eta(Y) X-2 \epsilon \eta(Y) \eta(X) \xi-B h(X, Y) .
$$

Interchanging $X$ and $Y$ in (26) we get

$$
\phi P \nabla_{Y} X=-A_{\phi X} Y-g(X, Y) \xi-\epsilon \eta(X) Y-2 \epsilon \eta(Y) \eta(X) \xi-B h(X, Y) .
$$

Substracting (27) from (26) we have

$$
\phi P[X, Y]=-A_{\phi Y} X+A_{\phi X} Y-\epsilon \eta(Y) X+\epsilon \eta(X) Y .
$$

Now since $M$ is $\xi$-vertical, $[X, Y] \in D^{\perp}$ iff

$$
A_{\phi X} Y-A_{\phi Y} X=\epsilon[\eta(Y) X-\eta(X) Y] .
$$

So the proof is complete.

D-umbilic (resp. $\mathrm{D}^{\perp}$-umbilic) contact CR-submanifold of indefinite Lorentzian para-Sasakian manifold is defined as follows:

Definition 3 A contact CR-submanifold $M$ of an indefinite Lorentzian paraSasakian manifold is said to be D-umbilic (resp. $\mathrm{D}^{\perp}$-umbilic) if $\mathrm{h}(\mathrm{X}, \mathrm{Y})=$ $\mathrm{g}(\mathrm{X}, \mathrm{Y}) \mathrm{L}$ holds for all $\mathrm{X}, \mathrm{Y} \in \mathrm{D}$ (resp. $\left.\mathrm{X}, \mathrm{Y} \in \mathrm{D}^{\perp}\right)$, $\mathrm{L}$ being some normal vector field. 
Contact CR-submanifolds of indefinite Lorentzian para-Sasakian manifold 163

In view of the above definition we state and prove the following proposition:

Proposition 3 Suppose $\mathrm{M}$ is a D-umbilic contact $C R$-submanifold of an indefinite Lorentzian para-Sasakian manifold $\tilde{M}$. If $\mathrm{M}$ is $\xi$-horizontal (resp. $\xi$ vertical) then $\mathrm{M}$ is $\mathrm{D}$-totally geodesic (resp. $\mathrm{D}^{\perp}$-totally geodesic).

Proof. Consider $M$ as D-umbilic $\xi$-horizontal contact CR-submanifold. Then we have from Definition 3

$$
h(X, Y)=g(X, Y) L \quad \forall X, Y \in D,
$$

L being some normal vector field on $M$. By putting $X=Y=\xi$ and using (19) we have

$$
h(\xi, \xi)=g(\xi, \xi) L
$$

i.e. $\quad L=0$,

and consequently we get $h(X, Y)=0$, which proves that $M$ is D-totally geodesic.

Similarly, it can be easily shown that if $M$ is $D^{\perp}$-umbilic $\xi$-vertical contact CR-submanifold then it is $\mathrm{D}^{\perp}$-totally geodesic.

\section{Mixed totally geodesic contact CR-submanifolds of indefinite Lorentzian para-Sasakian manifold}

In this section we define mixed totally geodesic contact CR-submanifolds of an indefinite Lorentzian para-Sasakian manifold (followed [12]).

Definition 4 A contact CR-submanifold $M$ of an indefinite Lorentzian paraSasakian manifold $\tilde{\mathrm{M}}$ is said to be mixed totaly geodesic if $\mathrm{h}(\mathrm{X}, \mathrm{Y})=0 \forall \mathrm{X} \in \mathrm{D}$ and $\mathrm{Y} \in \mathrm{D}^{\perp}$.

Then we extract the following lemma and theorem

Lemma 1 Let $M$ be a contact CR-submanifold of an indefinite Lorentzian para-Sasakian manifold. Then $\mathrm{M}$ is mixed totally geodesic iff

$$
\begin{array}{ll}
A_{N} X \in D, & \forall X \in D, \quad \text { and } \forall \text { normal vector field } N, \\
A_{N} X \in D^{\perp}, & \forall X \in D^{\perp} \text { and } \forall \text { normal vector field } N .
\end{array}
$$


Proof. If $M$ is mixed totally geodesic, then from (8), we get

$$
h(X, Y)=0,
$$

i.e., iff $A_{N} X \in D, \forall X \in D$ and $\forall$ normal vector field $N$. Conversely, if $M$ is mixed totally geodesic, then using (8) we easily observe that $A_{N} X \in D^{\perp}$, $\forall \mathrm{X} \in \mathrm{D}^{\perp}$ and $\forall$ normal vector field $\mathrm{N}$.

Hence the lemma is proved.

Using condition (29) we obtain the following theorem

Theorem 2 If $\mathrm{M}$ is a mixed totally geodesic contact CR-submanifold of an indefinite Lorentzian para-Sasakian manifold, then

$$
\begin{aligned}
& A_{\phi N} X=-\phi A_{N} X, \\
& \nabla_{X}^{\perp} \phi N=\phi \nabla_{X}^{\perp} N
\end{aligned}
$$

$\forall \mathrm{X} \in \mathrm{D}$ and $\forall$ normal vector field $\mathrm{N}$.

Proof. We get from (29), (6), (7) and after having some calculations we derive

$$
\begin{aligned}
& \nabla_{X} \phi N=\phi \nabla_{X}^{\perp} \mathrm{N}-\phi A_{N} X, \\
& \nabla_{X} \phi N=-A_{\phi N} X+\nabla_{X}^{\perp} \phi N .
\end{aligned}
$$

Comparing the above two equations we have the required theorem. Hence the proof follows.

Again we have the following definition

Definition 5 A contact CR-submanifold $\mathrm{M}$ of an indefinite Lorentzian paraSasakian manifold $\tilde{M}$ is called foliate contact $C R$-submanifold $\tilde{M}$ if $\mathrm{D}$ is involute. If $\mathrm{M}$ is a foliate $\xi$-horizontal contact CR-submanifold, we know from [3]

$$
h(\phi X, \phi Y)=h\left(\phi^{2} X, Y\right)=-h(X, Y) .
$$

Considering the above definition we give the following proposition.

Proposition 4 If $\mathrm{M}$ is a foliate $\xi$-horizontal mixed totally geodesic contact CR-submanifold $\mathrm{M}$ of an indefinite Lorentzian para-Sasakian manifold, then

$$
\phi A_{N} X=A_{N} \phi X
$$

for all $\mathrm{X} \in \mathrm{D}$ and normal vector field $\mathrm{N}$. 
Proof. From (21) and (8) we compute the following:

$$
g(h(X, \phi Y), N)=g\left(\phi A_{N} X, Y\right),
$$

i.e.

$$
g(h(\phi X, Y), N)=g\left(A_{N} \phi X, Y\right) .
$$

Therefore

$$
\phi A_{N} X=A_{N} \phi X
$$

Hence the proof follows.

\section{Anti-invariant distribution $\mathrm{D}^{\perp}$ on totally umbili- cal contact CR-submanifold of an indefinite Lorentzian para-Sasakian manifold}

Here we consider a contact CR-submanifold $M$ of an indefinite Lorentzian para-Sasakian manifold $\tilde{M}$. Then we establish the following theorem.

Theorem 3 Let $M$ be a totally umbilical contact CR-submanifold of an indefinite Lorentzian para-Sasakian manifold $\tilde{M}$. Then the anti invariant distribution $\mathrm{D}^{\perp}$ is one dimensional, i.e. $\operatorname{dimD}^{\perp}=1$.

Proof. For an indefinite Lorentzian para-Sasakian structure we have

$$
\left(\tilde{\nabla}_{Z} \phi\right) W=g(Z, W) \xi+\epsilon \eta(W) Z+2 \epsilon \eta(W) \eta(Z) \xi .
$$

Also by the covariant derivative of tensor fields (for any $Z, W \in \Gamma\left(D^{\perp}\right)$ we know

$$
\tilde{\nabla}_{Z} \phi W=\left(\tilde{\nabla}_{Z} \phi\right) W+\phi \tilde{\nabla}_{Z} W .
$$

Using (37), (38), (6), (7) and (4) we obtain

$$
\begin{aligned}
\nabla_{Z}^{\perp} \phi W-g(H, \phi W) Z= & \phi\left[\nabla_{Z} W+g(Z, W) H\right]+g(Z, W) \xi \\
& +\epsilon \eta(W) Z+2 \epsilon \eta(W) \eta(Z) \xi
\end{aligned}
$$

for any $\mathrm{Z}, \mathrm{W} \in \Gamma\left(\mathrm{D}^{\perp}\right)$.

Taking the inner product with $Z \in \Gamma\left(\mathrm{D}^{\perp}\right)$ in (39) we obtain

$$
\begin{aligned}
-g(H, \phi W)\|Z\|^{2}= & g(Z, W) g(\phi H, Z)+\epsilon \eta(W)\|Z\|^{2}+g(Z, W) g(\xi, Z) \\
& +2 \eta(W) \eta(Z) g(Z, \xi) .
\end{aligned}
$$


Using (2) after a brief calculation we have

$$
\begin{aligned}
g(H, \phi W)= & -\frac{g(Z, W) g(\phi H, Z)}{\|Z\|^{2}}-\frac{g(Z, W) g(\xi, Z)}{\|Z\|^{2}} \\
& -\epsilon g(W, \xi)-2 \frac{g(Z, \xi)^{2} g(W, \xi)}{\|Z\|^{2}}
\end{aligned}
$$

Interchanging $Z$ and $W$ we have

$$
\begin{aligned}
g(H, \phi Z)= & -\frac{g(Z, W) g(\phi H, W)}{\|W\|^{2}}-\frac{g(Z, W) g(\xi, W)}{\|W\|^{2}} \\
& -\epsilon g(Z, \xi)-2 \frac{g(W, \xi)^{2} g(Z, \xi)}{\|W\|^{2}} .
\end{aligned}
$$

Substituting (41) in (40) and simplifying we get

$$
\begin{aligned}
& g(H, \phi W)\left[1-\frac{g(Z, W)^{2}}{\|Z\|^{2}\|W\|^{2}}\right]-\frac{g(Z, W)}{\|Z\|^{2}}\left[\frac{g(Z, W) g(\xi, W)}{\|W\|^{2}}-g(Z, \xi)\right] \\
& -\epsilon\left[\frac{g(Z, W) g(\xi, Z)}{\|Z\|^{2}}-g(W, \xi)\right] \\
& -2 g(z, \xi) g(W, \xi)\left[\frac{g(Z, W) g(W, \xi)}{\|W\|^{2}\|Z\|^{2}}-\frac{g(Z, W)}{\|Z\|^{2}}\right]=0 .
\end{aligned}
$$

The equation (43) has a solution if $Z \| W$, i.e. $\operatorname{dim} D^{\perp}=1$.

Hence the theorem is proved.

Example 1 Let $\mathbf{R}^{3}$ be a 3-dimensional Euclidean space with rectangular coordinates $(x, y, z)$. In $\mathbf{R}^{3}$ we define

$$
\begin{gathered}
\eta=-d z-y d x \quad \xi=\frac{\partial}{\partial z} \\
\phi\left(\frac{\partial}{\partial x}\right)=\frac{\partial}{\partial y}, \quad \phi\left(\frac{\partial}{\partial y}\right)=\frac{\partial}{\partial x}-y \frac{\partial}{\partial z}, \quad \phi\left(\frac{\partial}{\partial z}\right)=0 .
\end{gathered}
$$

The Lorentzian metric $\mathrm{g}$ is defined by the matrix:

$$
\left(\begin{array}{ccc}
-\epsilon y^{2} & 0 & \epsilon y \\
0 & 0 & 0 \\
\epsilon y & 0 & -\epsilon
\end{array}\right)
$$


Contact CR-submanifolds of indefinite Lorentzian para-Sasakian manifold 167

Then it can be easily seen that $(\phi, \xi, \eta, g)$ forms an indefinite Lorentzian paraSasakian structure in $\mathbf{R}^{3}$ and the above results can be verified for this example.

\section{Acknowledgement}

This work is sponsored by UGC - BSR, UGC, India.

\section{References}

[1] A. Bejancu, CR-submanifolds of a Kaehlerian manifold I, Proc. Amer. Math. Soc., 69 (1978), 135-142.

[2] A. Bejancu, CR-submanifolds of a Kaehlerian manifold II, Trans. Amer. Math. Soc., 250 (1979), 333-345.

[3] A. Bhattacharyya, B. Das, Contact CR-submanifolds of an indefinite trans-Sasakian manifold, Int. J. Contemp. Math. Sci, 6 (26) (2011), 12711282

[4] A. Bejancu, K. L. Duggal, Real hypersurfaces of indefinite Kaehler manifolds, Int. J. Math. Math. Sci., 16 (3) (1993), 545-556.

[5] D. E. Blair, Contact manifolds in Riemannian Geometry, Lecture Notes in Mathematics, vol. 509, Springer Verlag, Berlin, (1976).

[6] C. Calin, I. Mihai, On a normal contact metric manifold, Kyungpook Math. J. 45 (2005), 55-65.

[7] B. Y. Chen, Geometry of submanifolds, M. Dekker, New York, (1973).

[8] B. Y. Chen, K. Ogiue, On totally real submanifolds, Trans. Amer. Math. Soc., 193 (1974), 257-266.

[9] K. L. Duggal, A. Bejancu, Lightlike submanifold of semi-Riemannian Manifolds and Applications, vol. 364 of Mathematics and its applications, Kluwer Academic Publishers, Dordrecht, The Netherlands, 1996.

[10] K. L. Duggal, B. Sahin, Lightlike submanifolds of indefinite Sasakian manifolds, Int. J. Math. Math. Sci., (2007), 1-22. 
[11] T. H. Kang, S. D. Jung, B. H. Kim, Lightlike hypersurfaces of indefinite Sasakian manifolds, Indian J. Pure Appl. Math., 34 (9) (2003), 1369 1380.

[12] M. Kobayashi, CR-submanifolds of a Sasakian manifold, Tensor, N. S., 35 (1981), 297-307.

[13] M. Kon, Invariant submanifolds in Sasakian manifolds, Math. Ann. 219 (1976), 277-290.

[14] G. D. Ludden, Submanifolds of cosymplectic manifolds, Jour. of Diff. Geom., 4 (1970), 237-244.

[15] K. Matsumoto, On Lorentzian para contact manifolds, Bull. Yamagata Univ. Nat. Sci., 12 (1989), 151-156.

[16] K. Matsumoto, I. Mihai, On certain transformation in a Lorentzian paraSasakian manifold, Tensor N. S., 47 (1968), 189-197.

[17] K. Ogiue, Differential geometry of Kaehler submanifolds, Adv. Math., 13 (1974).

[18] J. A. Oubiña, New classes of almost contact metric structures, Publ. Math. Debrecen, 32 (3-4), (1985), 187-193.

[19] R. Prasad, V. Srivastava, On $€$ Lorentzian para-Sasakian manifolds, Commun. Korean Math. Soc. 27 (2) (2012), 297-306.

[20] M. Tarafdar, A. Bhattacharya, On Lorentzian para-Sasakian manifolds, Steps in differential geometry, Proceedings of the Colloquium on Differential Geometry, 25-30 july 2000, Debrecen, Hungary, 343-348.

[21] K. Yano, M. Kon, Anti-invariant submanifolds, Marcel Dekker Inc., New York, (1976). 